\title{
Role of $\pi$-electron conjugation in determining the electrical responsive properties of polychlorinated biphenyls: a DFT based computational study
}

\author{
Rakesh Maity $^{1} \cdot$ Debkumar Mandal $^{1} \cdot$ Ajay Misra $^{1}$
}

Received: 4 June 2019 / Accepted: 17 January 2020 / Published online: 15 February 2020

(c) Springer Nature Switzerland AG 2020

\begin{abstract}
Global reactivity descriptors e.g. average polarizabilty $\left(a_{a v}\right)$, chemical hardness $(\eta)$, electrophilicity index $(\omega)$ of some donor-acceptor substituted polychlorinated biphenyl (PCBs) i.e. 2,2',5,5'-tetrachloro-1,1'-biphenyl (PCB-1a), 2,5,2',5'-Tetrachloro-4'-dimethylamino-biphenyl-4-carbonitrile (PCB-1b), Dimethyl-(2,5,2',5'-tetrachloro-4'-nitro-biphenyl4-yl)-amine (PCB-1C), 3,3',5,5'-tetrachloro-1,1'-biphenyl (PCB-2a), 3,5,3',5'-Tetrachloro-4'-dimethylamino-biphenyl-4carbonitrile (PCB-2b) and Dimethyl-(3,5,3',5'-tetrachloro-4'-nitro-biphenyl-4-yl)-amine (PCB-2c) were computed along the torsional potential of biphenyl ring. Density functional based hybrid functional CAM-B3LYP with 6-31G(d) basis were used to all the computational study. Out of the six compounds, variation of the global reactivity descriptors e.g. $a_{a v}, \eta, \omega$ as a function of torsional angle of biphenyl ring, three compounds are not in conformity with the respective optimum principle i.e. minimum polarizability, maximum hardness and minimum electrophilicity principle. In this present research article, we have raised this issue that apart from electronically stable conformation, localization of $\pi$-electron plays a major role in determining the optimum values of global reactivity descriptors, $a_{a v}, \eta \& \omega$. On the other hand computation of first average hyper polarizability $\left(\beta_{\mathrm{av}}\right)$ as a function of torsional angle shows that the variation of $\beta_{\mathrm{av}}$ are in good agreement with the optical gap as well as electronic spatial extent $\left(\left\langle R^{2}\right\rangle\right)$ of the conformers of all the six compounds.
\end{abstract}

Keywords Polarizability · First hyper-polarizability · Chemical hardness · Optical gap · Electronic spatial extent $\left(<R^{2}>\right)$

\section{Introduction}

Biphenyl is a very interesting molecule for several reasons. In the gaseous phase it exists in twisted conformation, where the conjugation between the phenyl rings is not operative. However, the two rings become coplanar in the crystalline state [1], indicating the change of conformation due to crystal packing force. Polychlorinated biphenyl (PCB), is a class of organo halogen compounds synthesized by the reaction of chlorine with biphenyl. Polychlorinated biphenyls (PCBs) are industrially important chemicals used in various applications requiring chemical stability and have now become widely dispersed. A typical mixture of PCBs may contain over 100 compounds and is a colourless, viscous liquid. The mixture is relatively insoluble in water, showing stability at high temperatures, and is a good dielectric (electrical insulator). Polychlorinated biphenyls (PCBs) are aromatic, synthetic chemicals and there is no natural abandance in the environment [2-5]. They consist of two linked benzene rings in which some or all of the

Electronic supplementary material The online version of this article (https://doi.org/10.1007/s42452-020-2068-x) contains supplementary material, which is available to authorized users.

Ajay Misra, ajay@mail.vidyasagar.ac.in | 'Department of Chemistry and Chemical Technology, Vidyasagar University, Midnapore, W.B 721 102, India. 
hydrogen atoms are substituted by chlorine atoms. PCBs are harmful persistent organic pollutants (POPs) which are toxic, persist in the environment, bio accumulate through the food chain and are harmful to human health and the environment [6-13]. Because of the low rotational energy barriers the phenyl torsion in biphenyl displays a unique dependence on the state of aggregation $[14,15]$. Hence, the electron affinity of PCBs is considered as an important parameter in understanding their toxic nature [16-18]. Accordingly the calculation of electron affinity of various PCBs has invoked attention in theoretical interests. An important property of PCBs are generally intern resistance towards both acids and alkalis and thermally stable. This made them useful as applications, including dielectric fluids in transformers and capacitors, heat transfer fluids, and lubricants [19]. PCBs are relatively insoluble in water, and the solubility decreases with increased chlorination. PCBs are also highly soluble in nonpolar organic solvents and are combustible liquids. The combustion products are more hazardous than the material itself. The numbering system for the PCBs is also shown below. Positions $2,2^{\prime}, 6$, and $6^{\prime}$ are called ortho positions, positions $3,3^{\prime}, 5$, and $5^{\prime}$ are called meta positions, and positions 4 and $4^{\prime}$ are called para positions. The benzene rings can rotate freely and the two extreme configurations are planar (the two benzene rings in the same plane) and the nonplanar in which the benzene rings are at a $90^{\circ}$ angle to each other. The degree of non-planarity is largely determined by the nature number of substituents in the ortho positions. The substitution in the ortho positions with larger chlorine atoms forces the phenyl rings to rotate out of the planar configuration. Three-dimensional structure-property correlations for the prediction of thermodynamic properties of PCBs like the enthalpy of vaporization and enthalpy of sublimation. Organic molecules having conjugated m-electron network are excellent option since they can be easily synthesised and modified chemically and have extremely fast switching time as well as can resist high intensity radiation. Nonlinear optical properties of a variety of push-pull phenylenes have been studied extensively. Recently, Arulmozhiraja et al. [9] using density functional theory calculations have obtained structure, potential energy, and torsional barriers for some selected polychlorinated biphenyls. Thus DFT, which has repeatedly been proven to be a reliable methodology, could be utilized to calculate reliable torsional barriers for PCBs [20-25].

As example, 2,2',5,5'-tetrachloro-1,1'-biphenyl (PCB-1a), 2,5,2',5'-Tetrachloro-4'-dimethylamino-biphenyl-4-carbonitrile (PCB-1b), Dimethyl-(2,5,2', 5'-tetrachloro-4'-nitro-biphenyl-4yl)-amine (PCB-1C), 3,3',5,5'-tetrachloro-1, 1'-biphenyl (PCB-2a), 3,5,3',5'-Tetrachloro-4'-dimethylamino-biphenyl-4-carbonitrile (PCB-2b) and Dimethyl-(3,5,3',5'-tetrachloro-4'-nitro-biphenyl4-yl)-amine (PCB-2c) have been investigated as a subject of many experimental and theoretical studies on conformational behaviour, torsional barrier and NLO properties. Two aromatic $\pi$-conjugated units connected together by a single bond, produce new composite molecules. Apart from the torsional barriers, the structure and potential energy curve associated with rotation about the $\mathrm{C}-\mathrm{C}$ single bond of a $\mathrm{PCB}$ are of fundamental research interest. In chlorinated biphenyls, this balance in interactions is still perturbed by the chlorine atoms, which influences the geometrical parameters of biphenyls, specifically the torsional angle between the phenyl rings. However, the torsional angle between two phenyl rings with ortho substitution is nearly $90^{\circ}$. PCBs are known to interact with the cellular components, and hence, the addition and the removal of an electron during the formation of the complex are significant events. The electron acceptance as well as electron removal to PCBs lead to changes in the torsional angle of PCBs and hence their geometry. Unlike the distinction between the principles based on general quantum theory, i.e. the Huckel rule, the Woodward-Hoffmann rules, and the maximum molecular valence principle there are some principles, e.g. the hard-soft acid-base principle, the maximum hardness principle (MHP) and minimum polarisability principle (MPP) are rooted in the density functional theory (DFT) [26, 27]. The MPP was formulated on the basis of the MHP and an inverse relationship between chemical hardness and polarisability was found.

Since the inception of these principles scientists are using these as global reactivity descriptors to study the global minima for any potential surfaces. Our present computational study shows that the variation of these parameters to locate global minimum are not the thumb rule, rather the nature and position of substitution play an important role in determining the global energy minimum conformer.

\subsection{Computation details}

Chemical hardness ( $\eta$ ) has been identified as a useful global reactivity index in atoms, molecules, and clusters. The theoretical definition of chemical hardness has been provided by DFT, as the second derivative of electronic energy with respect to the number of electrons $\mathrm{N}$, for a constant external potential $\mathrm{V}(\mathrm{r})$ [28].

$\eta=\left(\frac{\delta^{2} E}{\delta N^{2}}\right)_{v(r)}$

Operational schemes for the calculation of chemical hardness are based on a finite difference method and thus,

$\eta \approx \frac{1}{2}(I . P-E . A)$ 
The electrophilicity is an another reactivity descriptor which allows quantitative classification of the global electrophilic nature of a molecule within a relative scale. Parr et al. [29] proposed the electrophilicity index as a measure of energy lowering due to maximal electron flow between donor and acceptor. They defined electrophilicity index as,

$\omega=\mu^{2} / 2 \eta$

According to this definition, this index measures the propensity of chemical species to accept electrons. A good nucleophile is characterised by lower value of chemical potential $(\mu)$, electrophilicity index $(\omega)$ and conversely a good electrophile is characterised by a high value of $\mu, \omega$. This new reactive index measures the stabilisation in energy when the system acquires an additional electronic charge from the environment.

These global quantities, as well as the mean polarisability values $\left(a_{\mathrm{av}}\right)$, have been found useful and complementary tools for the description of chemical reactivity in connection with minimum polarisability and MHPs. Polarisabilities are calculated at a wavelength of $1064 \mathrm{~nm}$ corresponding to the Nd:YAG frequency, a wavelength used for many Hyper Rayleigh Scattering experiments [30-32].

The first hyperpolarizability [33] is calculated as

$$
\beta_{\text {vec }}(-2 \omega ; \omega, \omega)=\sqrt{ }\left(\beta_{x}^{2}+\beta_{y}^{2}+\beta_{z}^{2}\right)
$$

where $\beta_{i}=\frac{\sum\left(\beta_{i j}+\beta_{j i j}+\beta_{j i}\right)}{3} ; i=x, y, z j=x, y, z$

In Density functional theory (DFT), as it is used for computational chemistry, the hybrid functional B3LYP appears to offer the greatest contribution in terms of number of applications which has been published. However, it is unsuccessful is a number of important applications, (1) the polarizability of long chains, (2) excitations using time dependent theory (TDDFT) for Rydberg states, and (3) charge transfer (CT) excitations. Our computational study also reveals that DFT with B3LYP hybrid functional largely overestimate aav and $\beta a v$ value of the donor-acceptor substituted biphenyl. The reason for these failures is understood. At long range the exchange potential behaves as $-0.2 r^{-1}$ instead of exact value $-r^{-1}$. Even through, the potential is an improvement over the LDA and BLYP, where there is no $r^{-1}$ dependence in the potential. On the other hand $\mathrm{H}$. likura et al. [34] introduced new hybrid exchange correlation functional using the Coulomb-attenuating method (CAM-B3LYP). This functional is a hybrid functional with improved long range properties. Since the titled compounds in this present article are mostly charge transfer in character, we have used DFT based CAM-B3LYP hybrid functional with 6-31G(d) basis is an optimum set of computational method for the titled compounds.
All computations are carried out by using Gaussian 09 programs [35]. Polychlorobiphenyls are optimized by using coulomb-attenuated hybrid exchange-correlation functional, CAM-B3LYP, which includes a mixture of Hartree-Fock exchange and DFT exchange correlation. One split-valence basis sets, 6-31G-(d) are utilized for this purpose. Energy for all the six molecules as a function of tortional angle of phenyl ring are optimized by fixing the dihedral angle and the other co-ordinates are allowed to relax steps of D.A angle scan is taken $10^{\circ}$ and the enrgy of each steps are plotted as a function of D.A to get the potential energy curve for all the causes.

\section{Results and discussion}

In the following sections, we have discussed the structures, torsional barriers, potential energy curves and all other global reactivity parameters for the selected PCBs molecule.

The PCBs were grouped into two categories:

1. $\mathrm{PCBs}$ with two chlorine atoms at the $2,5,2^{\prime}, 5^{\prime}$ positions as 2,2',5,5'-tetrachloro-1, 1'-biphenyl (PCB-1a), 2,5,2',5'-Tetrachloro-4'-dimethylamino-biphenyl4-carbonitrile (PCB-1b) and Dimethyl- $\left(2,5,2^{\prime}, 5^{\prime}-\right.$ tetrachloro-4'-nitro-biphenyl-4-yl)-amine (PCB-1C).

2. $\mathrm{PCBs}$ with two chlorine atoms at the $3,5,3^{\prime}, 5^{\prime}$ positions as 3,3',5,5'-tetrachloro-1, 1'-biphenyl (PCB-2a), $3,5,3^{\prime}, 5^{\prime}$-Tetrachloro-4'-dimethylamino-biphenyl4-carbonitrile (PCB-2b) and Dimethyl- $\left(3,5,3^{\prime}, 5^{\prime}\right.$ tetrachloro-4'-nitro-biphenyl-4-yl)-amine (PCB-2C).

\subsection{Potential energy}

Variation of energy of [(PCB-1a), (PCB-1b) \& (PCB-1C)] and [(PCB-2a), (PCB-2b) \& (PCB-2C)] as a function of dihedral angle are shown in Fig. 1. It is observed from both the table (S1a, S1b, S1c) and Fig. 1, the conformers having dihedral angle in the range $60^{\circ}-90^{\circ}$ are the stable ones in case of PCB-1a, PCB-1b \& PCB-1c. It is the position of substitution of $\mathrm{Cl}$ atom at the $5^{\prime}$ position which makes these three conformers having D.A $60-90^{\circ}$ energetically more relaxed and stable.

On the other hand the variation of energy as a function of DA of the phenyl ring for (PCB-2a), (PCB-2b) \& (PCB-2c) as shown Fig. 1, illustrate that the conformer with $D A 40^{\circ}$ are the most stable and that at $90^{\circ}$ are the least stable. In this case, the steric hindrance of the $\mathrm{Cl}$ atoms has no role on the relative orientation of the phenyl rings and hence similar orientation of the phenyl rings $\left(40^{\circ}\right)$ as that of unsubstituted biphenyl $\left(40^{\circ}\right)$ are obtained in this series of molecules. 

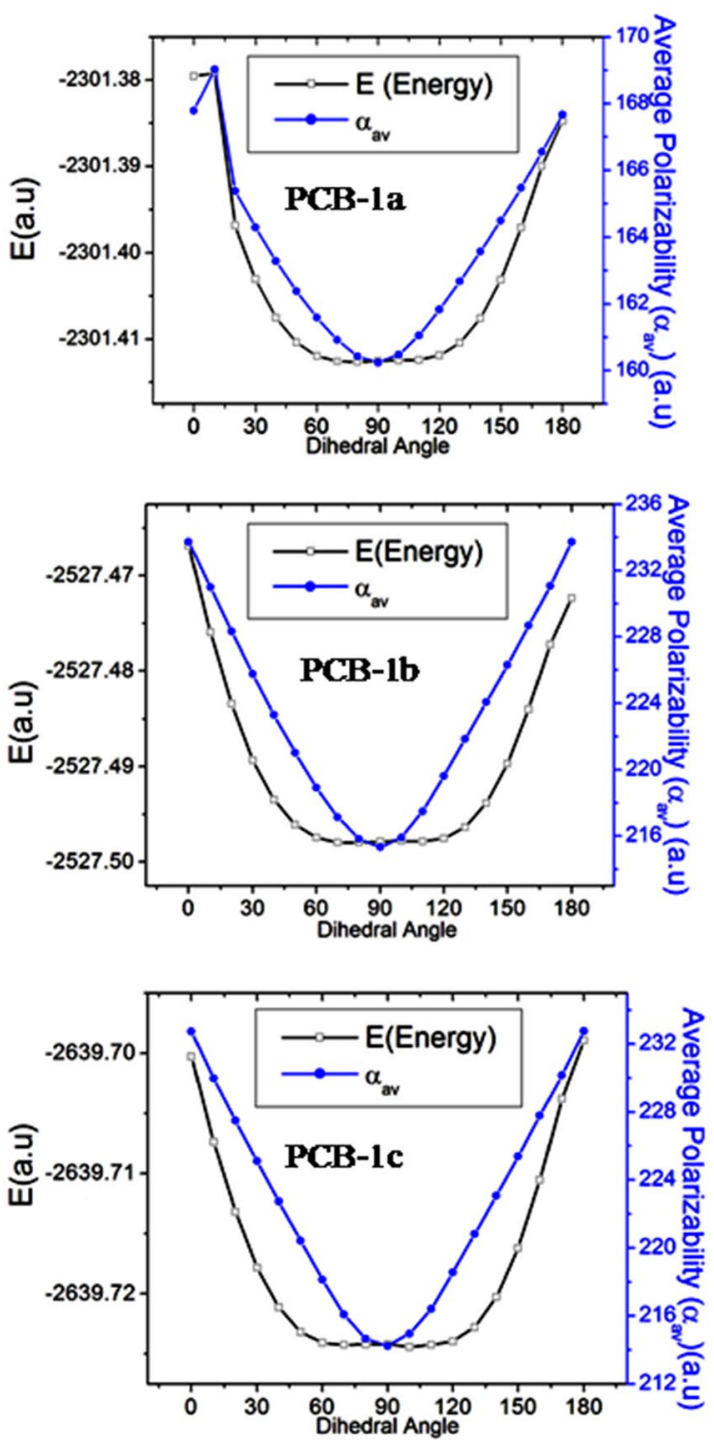
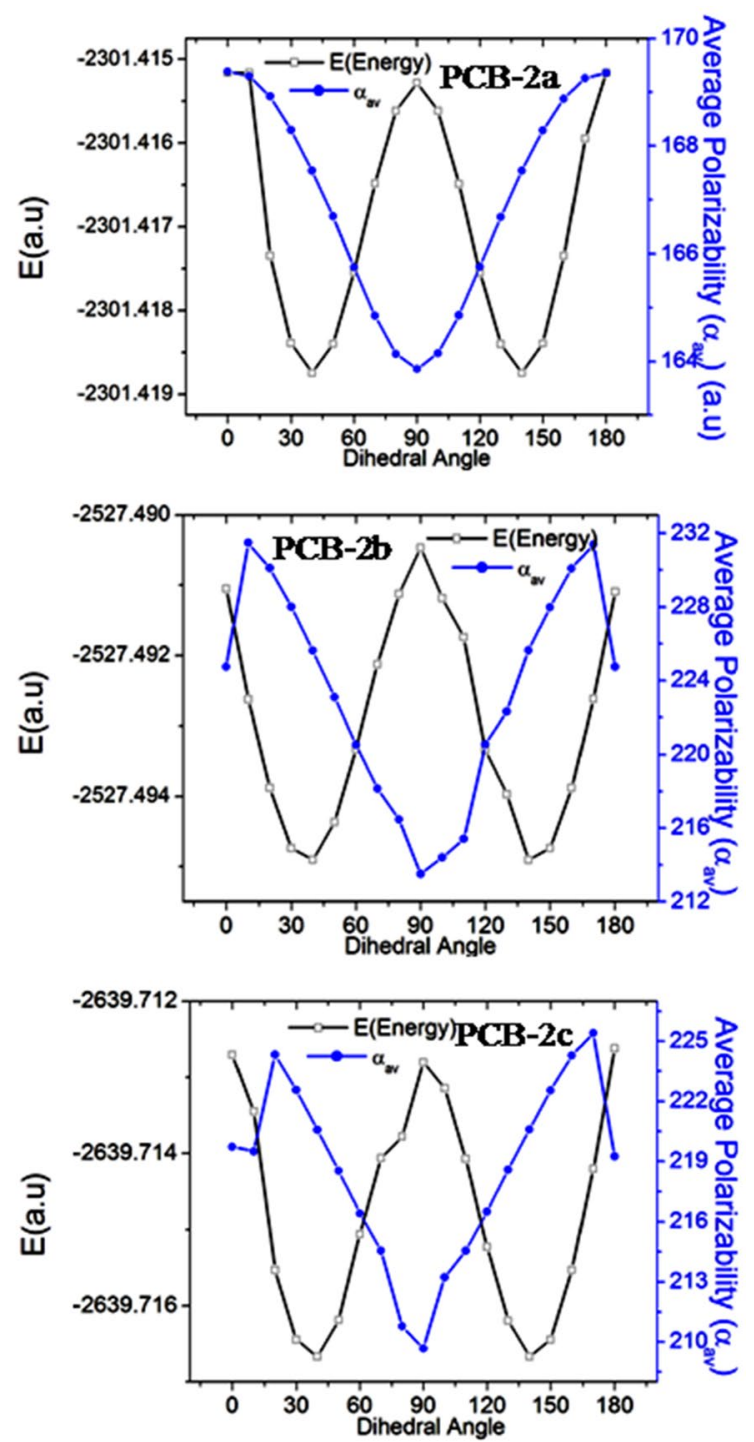

Fig. 1 Variation of average polarizability and energy versus dihedral angle of PCB-1a, PCB-1b, PCB-1c, PCB-2a, PCB-2b, PCB-2c

\subsection{Polarizability}

Variation of average polarizability $\left(a_{\mathrm{av}}\right)$ as a function of dihedral angle between the two phenyl ring of all the six compounds are shown in Fig. 1. It is observed that both the average polarizability $\left(a_{a v}\right)$ and energy of PCB-1a, PCB$1 b$ \& PCB-1c decreases as the DA varies from planer conformer and reaches their minimum value at $D A$ value $~ 90^{\circ}$. Hence the variations of $a_{a v}$ as a function dihedral angle are in conformity with the minimum polarizability principle. On the other hand, the minimum and maximum energy conformation for PCB-2b \& PCB-2c are observed at a dihedral value $40^{\circ}$ and $90^{\circ}$ respectively Fig. 1 and Table (S2a, S2b, S2c). According to MPP, $a_{a v}$ should have minimum value when the torsional angle between the two phenyl rings is $40^{\circ}$ and maximum value for perpendicular conformation. But the observed minimum and maximum value of average polarizability in this series of compounds are found at a dihedral angle $90^{\circ}$ and $40^{\circ}$ respectively. Thus this series of $\mathrm{PCBs}$ shows violation of minimum polarizability principle (MPP).

\subsection{Chemical hardness}

We also computed the chemical hardness ( $\eta$ ) of all the conformers, obtained as a function of dihedral angle (DA) between the two phenyl ring. Our computed chemical hardness as a function of dihedral angle are shown in Table-S1a,S1b,S1c \& Table-S2a,S2b,S2c and the plot of the same are shown in Fig. 2. Figure 2 illustrate that the value of chemical hardness of PCB-1a, PCB-1b \& PCB-1C are maximum where the energies have minimum value. 

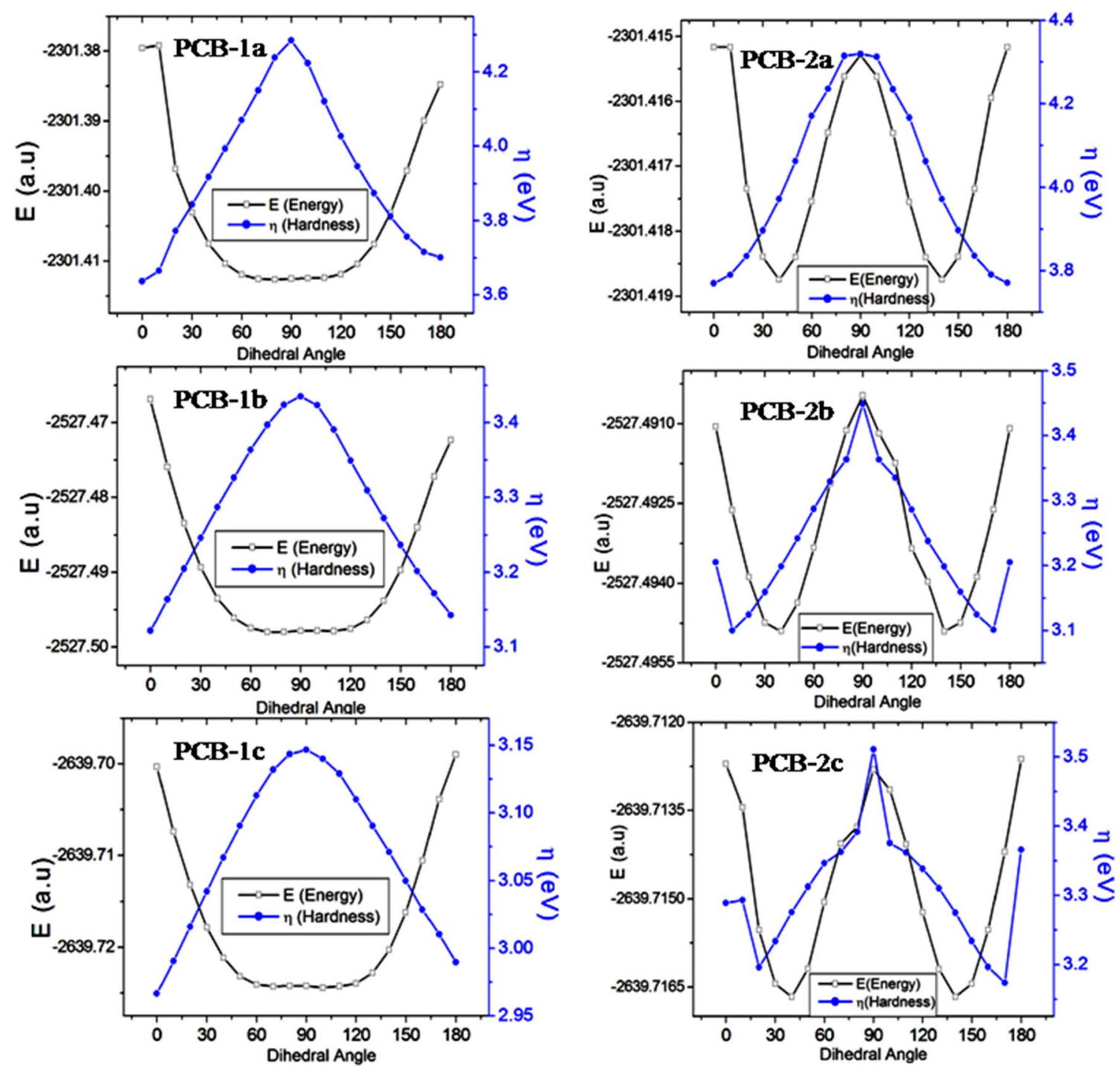

Fig. 2 Variation of Chemical hardness and energy versus dihedral angle of PCB-1a, PCB-1b, PCB-1c, PCB-2a, PCB-2b, PCB-2c

It is found that the $90^{\circ}$ conformers of this series of $\mathrm{PCB}$ has the minimum energy and maximum hardness value. Thus the variation ' $n$ ' as a function of dihedral angle between the phenyl rings are in conformity of the maximum hardness principle (MHP). On the other hand, the variation of chemical hardness ( $\eta$ ) of PCB-2a, PCB-2b \& $P C B-2 C$ as a function of dihedral angle between the two phenyl ring (Fig. 2) illustrate the maximum $\eta$ value at a D.A $90^{\circ}$, though the energy of this series of PCBs show minimum values at $D A 40^{\circ}$ and maximum energy at $90^{\circ}$. Thus this series of $\mathrm{PCBs}$ violates the maximum hardness principle (MHP).

\subsection{Electrophilicity index}

A good electrophile in this sense is characterised by a high value of chemical potential $(\mu)$ and a low value of hardness $(\eta)$. Variations of electrophilicity $(\omega)$ as a function of dihedral angle are shown in Fig. 3. In case of all the molecules, electrophilicity $(\omega)$ value decreases with the increase of dihedral angle, reaches minimum at the DA value $90^{\circ}$. It is interesting to note that high electrophilicity values have been obtained for the conformations corresponding to $\mathrm{DA}=180^{\circ}$ and $0^{\circ}$. The high electrophilicity value can be used as a criterion for the high toxic nature in the case of PCBs. It is clear that $\omega$ can be used as a proper descriptor for toxicity in $\mathrm{PCBs}$. We once again devide the PCBs into two class. In case of PCB-1a, PCB-1b \& PCB-1c, the value of electrophilicity index reaches minima at their minimum 

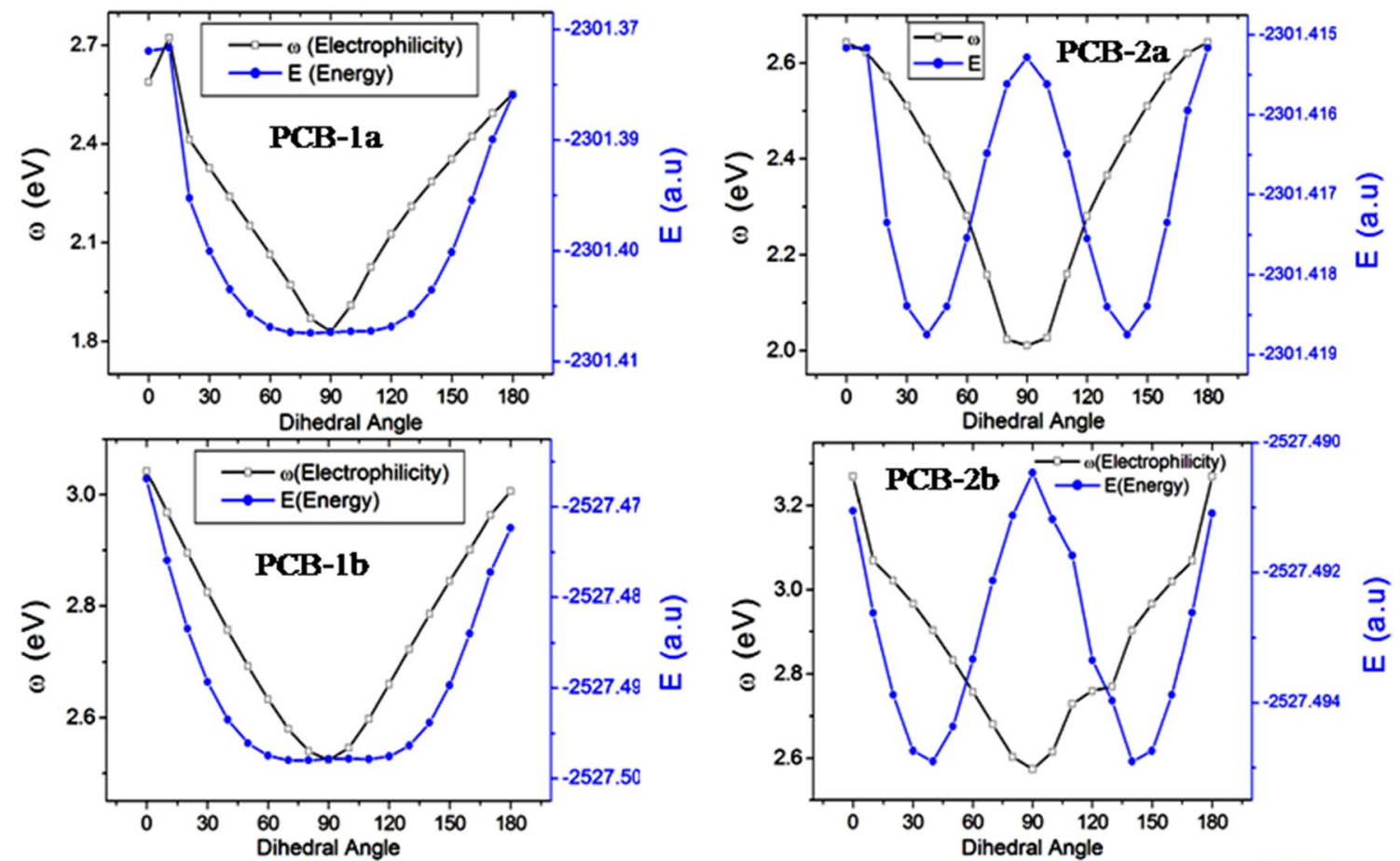

$-2527.47$
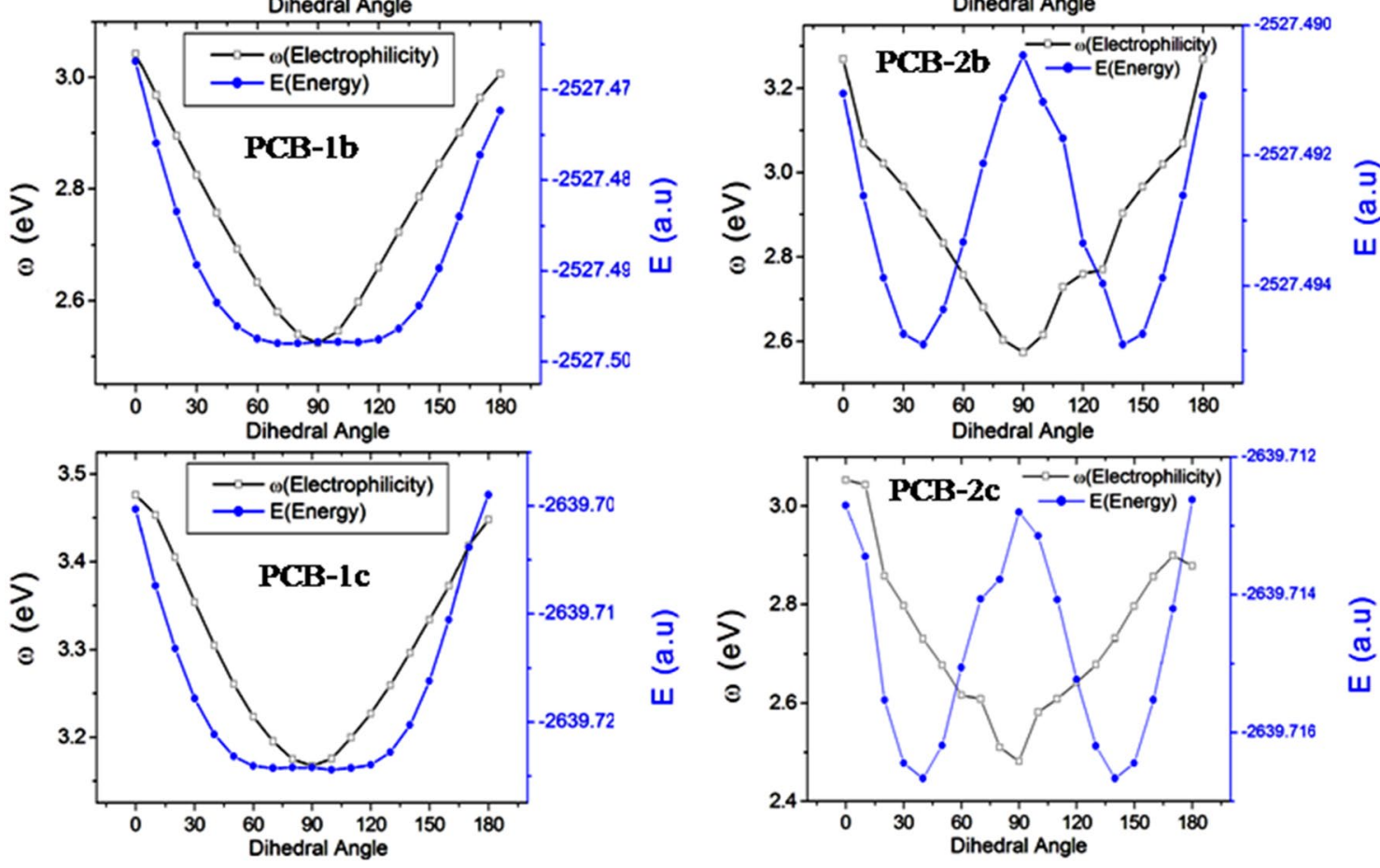

Fig. 3 Variation of Electrophilicity and energy versus dihedral angle of PCB-1a, PCB-1b, PCB-1c, PCB-2a, PCB-2b, PCB-2c

energy conformation i.e. at a dihedral angle value of $90^{\circ}$. Thus this series of PCBs supports the minimum electrophilicity principle as suggested by Par et al. [26] But the other series of PCB i.e. PCB-2a, PCB-2b \& PCB-2c have maximum energy conformer at $D A$ value $90^{\circ}$ and these conformers have lowest value of $\omega$ in their series. Hence these variations of $\omega$ are not in conformity of minimum electrophilicity principle.

\subsection{Global reactivity descriptors and $\pi$-electron density}

The above computational results clearly suggested that one series of $\mathrm{PCB}$, where the $\mathrm{Cl}$ atom at 2,2' position imposing strong steric hindrance are in conformity with the global reactivity descriptor as we pass from different conformers with the change in dihedral angle. But the other series where the $\mathrm{Cl}$ atoms at 3,3' \& 5,5' position, the most stable conformers have similar relative orientation as that unsubstituted biphenyl are not in conformity with 
Scheme 1 Structures and phenyl-phenyl $\mathrm{C}-\mathrm{C}$ bond length of Polychlorinated biphenyls

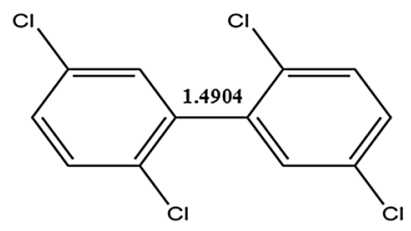

2,2',5,5'-tetrachloro-1,1'-biphenyl

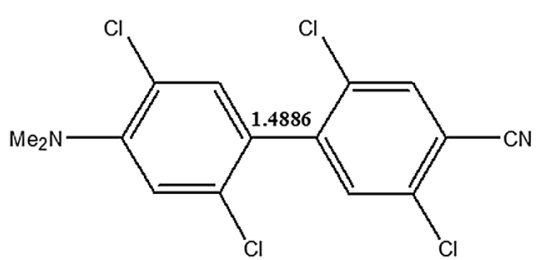

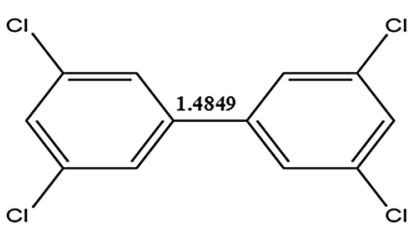

3,3',5,5'-tetrachloro-1,1'-biphenyl

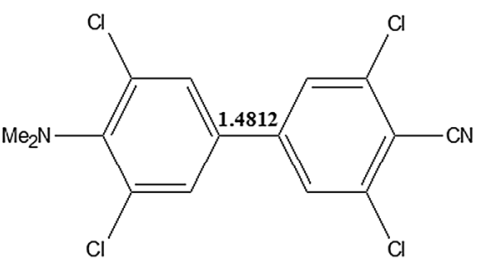

2,5,2',5'-Tetrachloro-4'-dimethylamino-biphenyl-4-carbon itrile 3,5,3',5'-Tetrachloro-4'-dimethylamino-biphenyl-4-carbonitrile
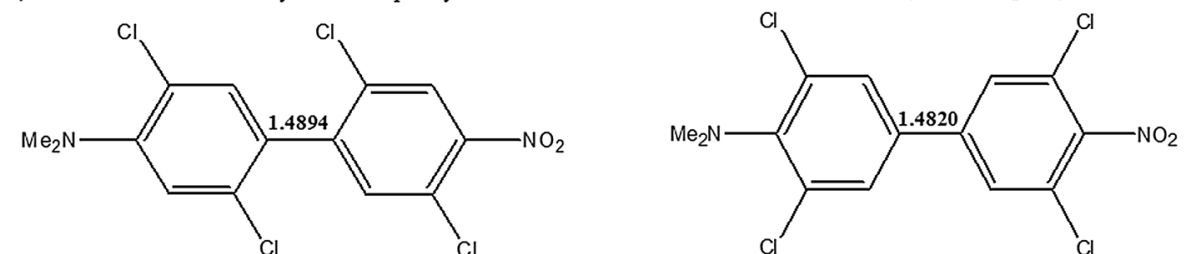

Dimethyl-(2,5,2',5'-tetrachloro-4'-nitro-biphenyl-4-yl)-amine Dimethyl-(3,5,3',5'-tetrachloro-4'-nitro-biphenyl-4-yl)-amine

the global reactivity descriptors as we vary the dihedral angle between the two phenyl ring. Interestingly except energy, the variation of all the global reactivity descriptors as a function of dihedral angle show similar trend. Maximum values of chemical hardness ( $\eta$ ) are observed at a DA $90^{\circ}$ for all the six PCBs studied in this article. On the other hand, the minimum value of average polarizability $\left(a_{\mathrm{av}}\right)$ and electrophilicity $(\omega)$ are observed at DA value of $90^{\circ}$ to all the PCBs.

Orientations of phenyl rings in their energy optimized conformers of these two series of molecules are due to extended conjugation through bi-phenyl ring. In case of PCB-1a, PCB-1b \& PCB-1c, the two phenyl ring will prefer to minimize the steric repulsion of the $\mathrm{Cl}$ atoms at $2,2^{\prime}$ position by rotating the phenyl rings with respect to each other by $90^{\circ}$. On the other hand, the other series of PCBs i.e. PCB-2a, PCB-2b, PCB-2c, there is no such strong steric repulsion due to $\mathrm{Cl}$ atom. Here the steric repulsion is due to $\mathrm{H}$-atom is much less compare to $\mathrm{Cl}$ at the $2,2^{\prime}$ position and the phenyl rings orients with respect to each other by $40^{\circ}$ to minimize the energy. At this $40^{\circ}$ orientation, there is still possibility of partial overlap of the $p_{z}$ orbitals of the adjacent carbon atom of the two phenyl ring. This partial conjugation of $\pi$-electron between the two phenyl ring makes the $40^{\circ}$ orientation of the PCBs of this series as stable one. In this connection, we have computed the $\mathrm{C}-\mathrm{C}$ bond distance between the adjacent carbon atom of all the PCBs studied here. Our computed results as given in Scheme 1 shows that the PCBs in the series PCB-2a, PCB-2b, PCB-2c has shorter $C-C\left(\sim 1.48 A^{\circ}\right)$ distance compare to the series PCB-1a, $\mathrm{PCB}-1 \mathrm{~b}$ \& $\mathrm{PCB}-1 \mathrm{c}$ where $\mathrm{C}-\mathrm{C}$ distance is $\sim 1.49 \mathrm{~A}^{\circ}$. Thus the $\pi$-electron conjugation over the two phenyl ring play a major role in determining the $40^{\circ}$ conformation as the stable form of PCBs in the series, PCB-2a, PCB-2b, PCB-2c.

HOMO-LUMO electron densities of all the six PCBs are shown in Fig. S1. HOMO electron distribution for $90^{\circ} \mathrm{con}-$ formations of all the PCBs show that $\pi$-electrons are localized within one phenyl rings. But at $40^{\circ}$ conformations, $\pi$-electrons are distributed over the two phenyl ring for all PCBs as shown in Fig. S1. This localization of $\pi$-electrons within a single phenyl ring makes the $90^{\circ}$ conformation less polarisable, harder and less electrophilic for all the PCBs. Therefore the localization of $\pi$-electrons results the optimum values of all the global reactivity descriptors at their $90^{\circ}$ conformation. 

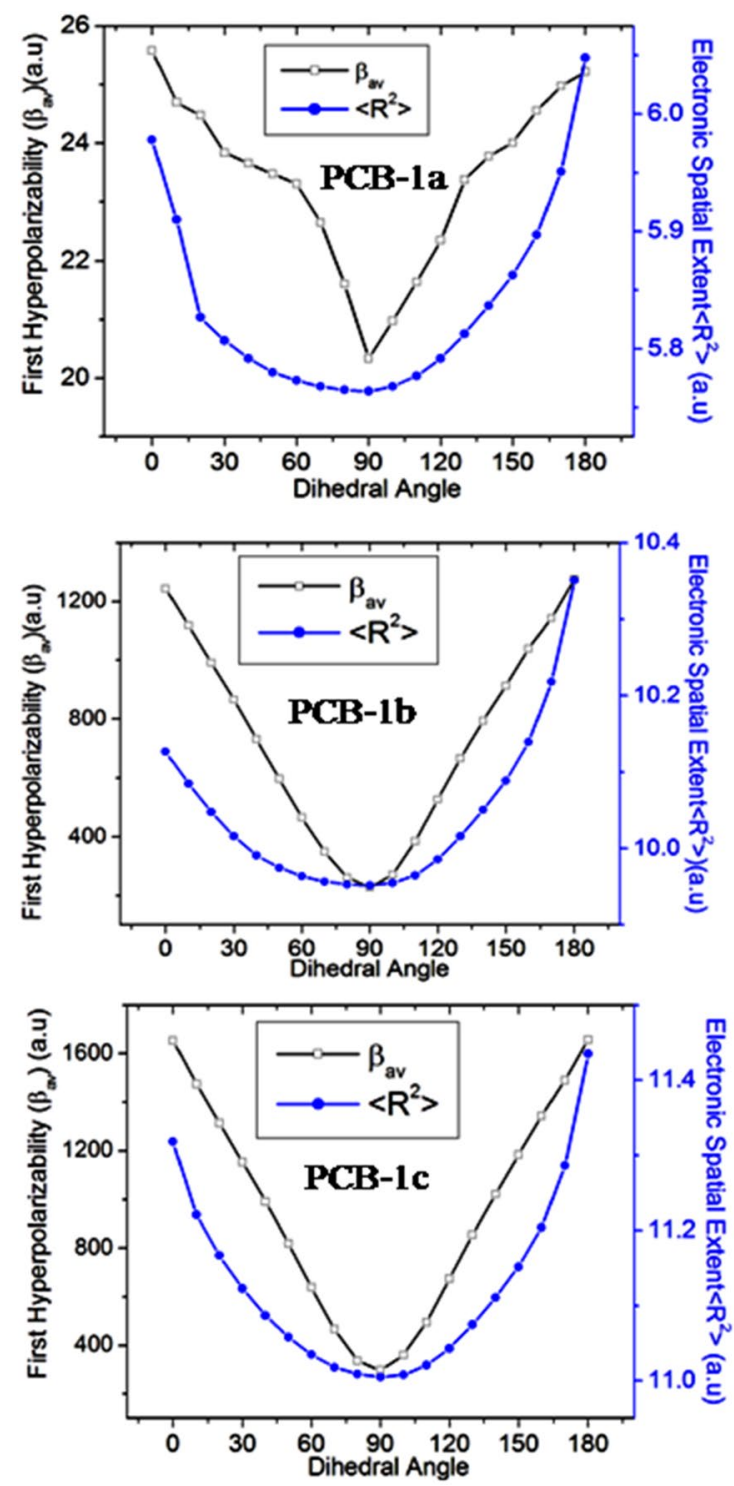
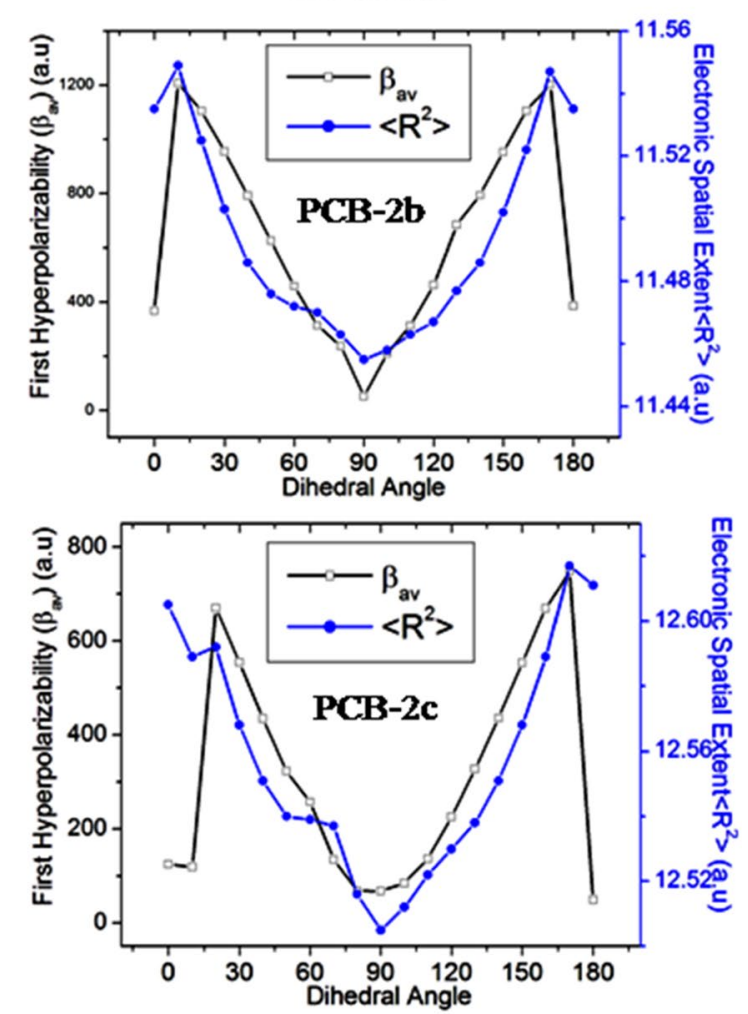

Fig. 4 Variation of First Hyper polarizability $\left(\beta_{\mathrm{av}}\right)$ and electronic spatial extent $\left.\left(<R^{2}\right\rangle\right)$ versus dihedral angle of PCB-1a, PCB-1b, PCB-1c, PCB2a, PCB-2b, PCB-2c

\subsection{First hyper polarizability}

Variations of first hyper polarizability $\left(\beta_{\mathrm{av}}\right)$ as a function of torsional angle between the two phenyl rings of all the PCBs molecules are shown in Fig. 4. Maximum values of first hyper polarizability for all the compounds are obtained when the torsional angle in $0^{\circ}$ or $180^{\circ}$, i.e. the planer conformation of biphenyl ring. $\beta_{\mathrm{av}}$ for both the series of molecule decreases with the increase of dihedral angle value and reaches the minimum at a dihedral value of $90^{\circ}$. In case of all the PCBs the variations of $\beta_{\mathrm{av}}$ as a function of dihedral angle are inversely proportional to the optical gap $\left(S_{1}-S_{0}\right)$ (Table $-S 1 a, S 1 b, S 1 c \& S 2 a$, $\mathrm{S} 2 \mathrm{~b}, \mathrm{~S} 2 \mathrm{c}$ ). The effect of substituent (donor-acceptor) on 

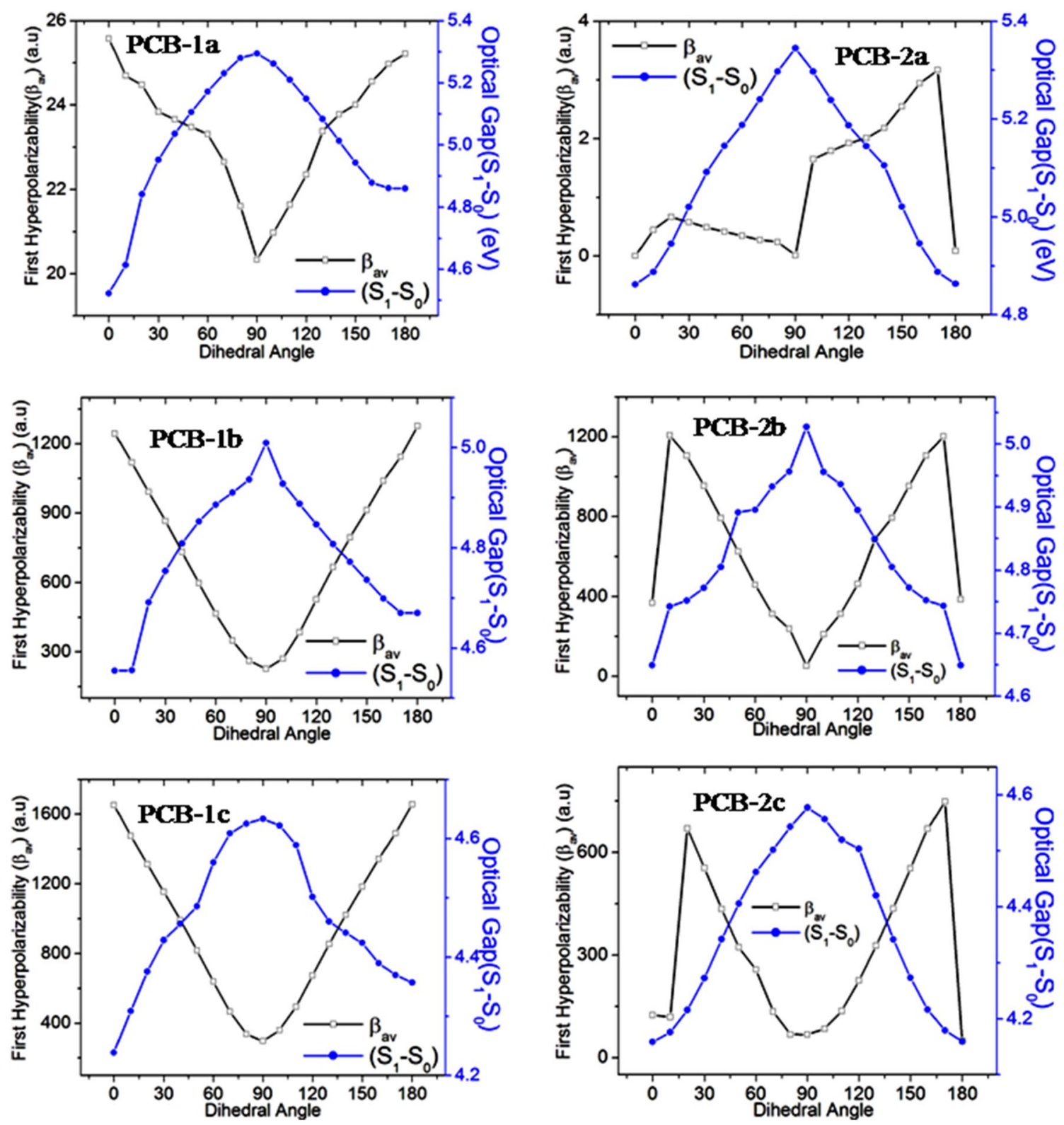

Fig. 5 Variation of First Hyper polarizability and Optical Gap $\left(\mathrm{S}_{1}-\mathrm{S}_{0}\right)$ versus dihedral angle of PCB-1a, PCB-1b, PCB-1c, PCB-2a, PCB-2b, PCB-2c

NLO response of the PCBs compounds and its effect on the optical gap were highlighted (Fig. 5 and Table-S3). Figure 5 illustrates that the extent of $\pi$ conjugation is maximum i.e. optical gap has minimum value for planer conformation of all the PCBs and less $\pi$ conjugation or maximum optical gap is found for the conformations having two phenyl group perpendicular to each other i.e. DA value of $90^{\circ}$. Hence for all the PCBs in this series show maximum $\beta_{\mathrm{av}}$ at $\mathrm{DA}$ value $0^{\circ}$ and minimum value at $D A$ value $90^{\circ}$.

We also computed electronic spatial extent $\left(<R^{2}>\right)$ which is a measure of extent of electronic volume over the molecule, as a function of dihedral angle. Figure 4 (Table-S1a, S1b, S1c \& Table- S2a, S2b, S2c) clearly suggests that the planer conformations of all the six PCBs shows higher $\left\langle R^{2}>\right.$ value and it decreases with the increase $D A$ and reaches its minimum value at DA $90^{\circ}$ i.e. when the two phenyl ring are perpendicular to each other. Our previous computational study suggests that higher the value of $\left\langle R^{2}\right\rangle$, higher is the value of $\beta_{\mathrm{av}}$ [36]. Again, the $\beta_{\mathrm{av}}$ value is directly proportional to the extent of charge transfer character of the molecule i.e. smaller the optical gap $\left(S_{1}-S_{0}\right)$, higher is the charge transfer character and higher is the $\beta_{\text {av }}$ value. Thus the variation of optical gap $\left(S_{1}-S_{0}\right)$ and 


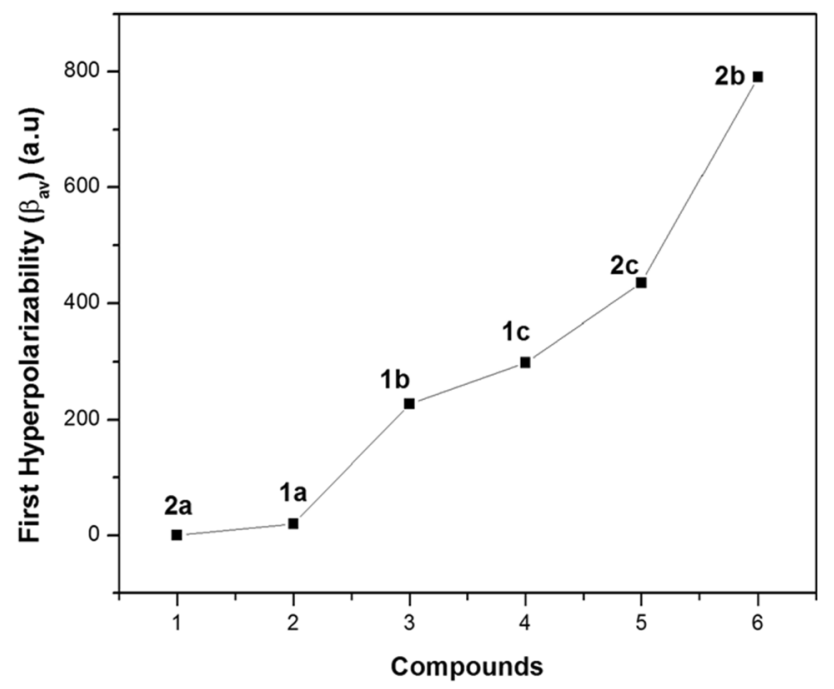

Fig. 6 Variation of First Hyper polarizability $\left(\beta_{\text {av }}\right)$ versus all the PCBs (PCB-1a, PCB-1b, PCB-1c, PCB-2a, PCB-2b, PCB-2c)

electronic spatial extent $\left.\left(<R^{2}\right\rangle\right)$ explain the variation of $\beta_{\mathrm{av}}$ of all the PCBs as a function of dihedral angle between the two phenyl ring.

Since the first hyper polarizability is a measure of nonlinear optical property of the molecule, we have compared the $\beta_{\mathrm{av}}$ values of our studied PCBs at their minimum energy conformations and it is shown in Fig. 6. Our study revealed that the donor acceptor containing $\mathrm{PCBs}$ showing maximum charge transfer character within the molecule has the higher $\beta_{\mathrm{av}}$ value.

\section{Conclusions}

Electrical responsive parameters like polarisabilty $\left(a_{a v}\right)$, chemical hardness $(\eta)$, electrophilicity $(\omega)$ and first hyper polarizability $\left(\beta_{\mathrm{av}}\right)$ of donor-acceptor substituted polychlorinated biphenyl 2,2',5,5'-tetrachloro-1,1'-biphenyl (PCB-1a), 2,5,2', 5'-Tetrachloro-4'-dimethylaminobiphenyl-4-carbonitrile (PCB-1b), Dimethyl- $\left(2,5,2^{\prime}, 5^{\prime}-\right.$ tetrachloro-4'-nitro-biphenyl-4-yl)-amine (PCB-1C), 3,3',5,5'-tetrachloro-1, 1'-biphenyl (PCB-2a), 3,5,3',5'-Tetrachloro4'-dimethylamino-biphenyl-4-carbonitrile (PCB-2b) and Dimethyl-(3,5,3',5'-tetrachloro-4'-nitro-biphenyl-4-yl)amine (PCB-2C) have been carried out as a function of torsional angle of the two phenyl rings using DFT-based theory. Here, the six PCBs are devided into two series. In series one the $\mathrm{Cl}$ atoms are present in the $2,2^{\prime}, 5,5^{\prime}$ position of the phenyl ring i.e. PCB-1a, PCB-1b, PCB-1c and on the other series the $\mathrm{Cl}$ atom present at $3,3^{\prime}, 5,5^{\prime}$ position. Our computed global reactivity descriptors, $\eta, a_{a v}, \omega$ are in conformity with MPP, MHP \& MEP for series one PCB. On the other hand the other series of PCBs i.e. PCB-2a, PCB-2b,
PCB-2c do not obey the MPP, MHP \& MEP as we vary the dihedral angle between the two phenyl rings. This apparent violation of the optimum principle related to global reactivity descriptors have been explained due to partial overlap of $p_{z}$ orbitals of the two adjacent $C$-atoms which makes the $40^{\circ}$ conformer more stable than the $90^{\circ}$ conformer. We also computed first hyper polarizability $\left(\beta_{\mathrm{av}}\right)$, electronic spatial extent $\left.\left(<R^{2}\right\rangle\right)$ and optical gap $\left(S_{1}-S_{0}\right)$ for all the PCBs as a function of dihedral angle. This variation of $\beta_{\mathrm{av}}$ has nicely been explained with the change of $\left\langle R^{2}>\right.$ and optical gap as we vary the dihedral angle.

Acknowledgements R. Maity thanks to UGC-BSR for his individual research fellowship. Departmental computational facilities from DST-FIST (Ref. No. SR/FST/CSI-235/2011) and UGC-SAP (Ref. No. F.59/2015/DRS-11 (SAP-11) programs are gratefully acknowledged. We are thankful to USIC, Vidyasagar University for providing Gaussian-09 software.

\section{Compliance with ethical standards}

Conflict of interest The authors declare that they have no conflict of interest.

\section{References}

1. Charbonneau GP, Delugeard $Y$ (1976) Structural transition in polyphenyls. III. Crystal structure of biphenyl at $110 \mathrm{~K}$. Acta Crystallogr Sect B 32:1420

2. Safe SH (1994) J Crit Rev Toxicol 24:87

3. Ballschmiter K, Zell M, Fresenius Z (1980) Analysis of polychlorinated biphenyls $(\mathrm{PCB})$ by glass capillary gas chromatography. Anal Chem 302:20

4. Safe S, Hutzinger O (1984) Polychlorinated biphenyls (PCBs) and polybrominated biphenyls (PBBs): biochemistry, toxicology, and mechanism of action. J Crit Rev Toxicol 13:319

5. Carpenter DO (2006) Polychlorinated biphenyls (PCBs): routes of exposure and effects on human health. Rev Environ Health 21:1

6. Van den Berg M, Birnbaum L, Bosveld AT, Brunstrom B, Cook P, Feeley M, Giesy JP, Hanberg A, Hasegawa R, Kennedy SW, Kubiak T, Larsen JC, van Leeuwen FX, Liem AK, Nolt C, Peterson RE, Poellinger L, Safe S, Schrenk D, Tillitt D, Tysklind M, Younes M, Waern F, Zacharewski T (1998) Toxic equivalency factors (TEFs) for PCBs, PCDDs, PCDFs for humans and wildlife. Environ Health Perspect 106:775

7. Oakley GG, Devanaboyina US, Robertson LW, Gupta RC (1996) Oxidative DNA damage induced by activation of polychlorinated biphenyls (PCBs): implications for PCB-induced oxidative stress in breast cancer. Chem Res Toxicol 9:1285

8. Erickson MD (1986) Analytical chemistry of PCBs. Butterworth Publishers, Boston

9. Arulmozhiraja S, Christopher Selvin P, Fujii T (2002) Structures, potential energy curves, and torsional barrier heights for selected polychlorinated biphenyls: a density functional theory study. J Phys Chem A 106:1765

10. Silberhorn EM, Glauert HP, Robertson LW (1990) Critical reviews in: carcinogenicity of polyhalogenated biphenyls: PCBs and PBBs. CRC Crit Rev Toxicol 20:439 
11. Wolff MS, Toniolo PG, Lee EW, Rivera M, Dubin N (1993) Blood levels of organochlorine residues and risk of breast cancer. J Natl Cancer Inst 85:648

12. Krieger N, Wolff MS, Hiatt RA, Rivera M, Vogelman J, Orentreich $N$ (1994) Breast cancer and serum organochlorines: a prospective study among white, black, and Asian women. J Natl Cancer Inst 86:589

13. Wassermann $M$, Nogueira DP, Tomatis L, Mirra AP, Shibata $H$, Arie G, Cucos S, Wassermann D (1976) Organochlorine compounds in neoplastic and adjacent apparently normal breast tissue. Bull Environ Contam Toxicol 15:478

14. Bastiansen O, Samdal S (1985) Structure and barrier of internal rotation of biphenyl derivatives in the gaseous state: Part 4. Barrier of internal rotation in biphenyl, perdeuterated biphenyl and seven non-ortho-substituted halogen derivatives. J Mol Struct 128:115

15. Carreira LA, Towns TG (1977) Raman spectra and barriers to internal rotation: biphenyl and nitrobenzene. J Mol Struct 41:1

16. Christopher RC, Davis DD, Habeck AE, Roy NK, Wirgin I (2012) Toxic effects of PCB126 and TCDD on shortnose sturgeon and Atlantic sturgeon. Environ Toxicol Chem 31:2324

17. Robertson LW, Hansen LG (2015) PCBs: recent advances in environmental toxicology and health effects. University Press of Kentucky, Lexington

18. Robertson LW, Ludewig G (2011) Polychlorinated Biphenyl (PCB) carcinogenicity with special emphasis on airborne PCBs. Gefahrst Reinhalt Luft 71:25

19. Afghan BK, Chau ASY (1989) Analysis of trace organics in the aquatic environment. CRC Press, Boca Raton

20. Arulmozhiraja S, Fujii T (2001) Torsional barrier, ionization potential, and electron affinity of biphenyl-a theoretical study. J Chem Phys 115:10589

21. Parthasarathi R, Padmanabhan J, Subramanian V, Maiti B, Chattaraj PK (2003) Chemical reactivity profiles of two selected polychlorinated biphenyls. J Phys Chem A 107:10346

22. Biedermann PU, Schurig V, Agranat I (1997) Enantiomerization of environmentally significant overcrowded polychlorinated biphenyls (PCBs). Chirality 9:350

23. Cullen JM, Kaiser EKL, QSAR in Environmental Toxicology, pp 39-66

24. Tang T-H, Nowakowska M, Guillet JE, Csizmadia IG (1991) Rotational barriers for selected polyfluorobiphenyl (PFB), polychlorobiphenyl (PCB) and polybromobiphenyl (PBB) congeners. J Mol Struct (Theochem) 232:133

25. Dorofeeva OV, Moiseeva NF, Yungman VS (2004) Thermodynamic properties of polychlorinated biphenyls in the gas phase. J Phys Chem A 108:8324

26. Pan S, Solà M, Chattaraj PK (2013) On the validity of the maximum hardness principle and the minimum electrophilicity principle during chemical reactions. J Phys Chem A 117:1843

27. Ghanty TK, Ghosh SK (1993) Correlation between hardness, polarizability, and size of atoms, molecules, and clusters. J Phys Chem 97:4951
28. Parr RG, Chattaraj PK (1991) Principle of maximum hardness. J Am Chem Soc 113:1854

29. Parr RG, Yang W (1984) Density functional approach to the frontier-electron theory of chemical reactivity. J Am Chem Soc 106:4049

30. Beg H, Das D, Ash S, Misra A (2013) Computation of polarizability, hyper-polarizability and hardness as descriptor for enol-keto tautomerizations of 2-hydroxy pyridines. Comput Theor Chem 1017:200

31. Mandal D, Maity R, Dey S, Misra A (2019) Effect of chain length and donor-acceptor substitution on the electrical responsive properties of conjugated biphenyls: a DFT-based computational study. Mol Phys 23:117

32. Kenis PJA, Noordman OFJ, Houbrechts S, Hummel GJV, Harkema S, Van Veggel FCJM, Clays K, Engbersen JFJ, Persoons A, Hulst NFV, Reinhoudt DN (1998) Second-order nonlinear optical properties of the four tetranitrotetrapropoxycalix [4] arene conformers. J Am Chem Soc 120:7875

33. Wang CH, Na-Na Ma, Sun XX, Sun SL, Qiu YQ, Liu PJ (2012) Modulation of the second-order nonlinear optical properties of the two-dimensional pincer Ru (II) complexes: substituent effect and proton abstraction switch. J Phys Chem A 116:10496

34. likura $\mathrm{H}$, Tsuneda T, Yanai T, Hirao K (2001) A long-range correction scheme for generalized-gradient-approximation exchange functionals. J Chem Phys 115:3540

35. Frisch MJ, Trucks GW, Schlegel HB, Scuseria GE, Robb MA, Cheeseman JR, Montgomery JA, Vreven Jr. T, Kudin KN, Burant JC,.Millam JM, lyengar SS, Tomasi J, Barone V, Mennucci B, Cossi M, Scalmani G, Rega N, Petersson GA, Nakatsuji H, Hada M, Ehara M, Toyota K, Fukuda R, Hasegawa J, Ishida M, Nakajima T, Honda Y, Kitao O, Nakai H, Klene M, Li X, Knox JE, Hratchian HP, Cross JB, Adamo C, Jaramillo J, Gomperts R, Stratmann RE, Yazyev O, Austin AJ, Cammi R, Pomelli C, Ochterski JW, Ayala PY, Morokuma K, Voth GA, Salvador P, Dannenberg JJ, Zakrzewski VG, Dapprich S, Daniels AD, Strain MC, Farkas O, Malick DK, Rabuck AD, Raghavachari K, Foresman JB, Ortiz JV, Cui Q, Baboul AG, Clifford S, Cioslowski J, Stefanov BB, Liu G, Liashenko A, Piskorz $P_{1}$ Komaromi I, Martin RL, Fox DJ, Keith T, Al-Laham MA, Peng CY, Nanayakkara A, Challacombe M, Gill PMW, Johnson B, Chen W, Wong MW, Gonzalez C, Pople JA (2009) Gaussian 09 (revision A.02), Gaussian Inc., Wallingford, CT

36. Scuderi D, Paladini A, Satta M, Catone D, Piccirillo S, Speranza M, Guidoni AG (2002) Chiral aggregates of indan-1-ol with secondary alcohols and water: laser spectroscopy in supersonic beams. Phys Chem Chem Phys 4:4999

Publisher's Note Springer Nature remains neutral with regard to jurisdictional claims in published maps and institutional affiliations. 